\title{
Prior Meditation Practice Modulates Performance and Strategy Use in Convergent- and Divergent-Thinking Problems
}

\author{
Lorenza S. Colzato • Ayca Szapora • Dominique Lippelt • \\ Bernhard Hommel
}

Published online: 29 October 2014

(C) Springer Science+Business Media New York 2014

\begin{abstract}
Scientific interest in meditation has significantly grown in the past years; however, so far, science has neglected the idea that different types of meditations may drive specific cognitive-control states. It has been shown that focusedattention (FA) and open-monitoring (OM) meditation exert specific effect on creativity; OM meditation induces a control state that promotes divergent thinking, a style of thinking that allows many new ideas to be generated, while FA meditation tends to support convergent thinking, the process of generating one possible solution to a particular problem. In the present study, by using creativity tasks tapping into convergent (compound Remote Associates Task; cRAT) and divergent thinking (Alternate Uses Task; AUT), we investigated whether this effect was modulated by prior meditation experience, by comparing a group of practitioners $(n=20)$ and a group of novices $(n=20)$. The enhancing effect of OM meditation on divergent thinking was found to be robust irrespective of prior experience. However, while solving convergentthinking problems, practitioners used an insight strategy, as opposed to an analytical approach, significantly more often than the novices.
\end{abstract}

Keywords Open monitoring · Focused attention - Creativity · Convergent thinking $\cdot$ Divergent thinking

\section{Introduction}

Like many others, Steve Jobs had often referred to meditation as the main source of his creativity (Isaacson 2011). When

L. S. Colzato $(\bowtie) \cdot$ A. Szapora $\cdot$ D. Lippelt $\cdot$ B. Hommel Institute for Psychological Research \& Leiden Institute for Brain and Cognition, Leiden University, Wassenaarseweg 52,

Leiden, The Netherlands

e-mail: colzato@fsw.leidenuniv.nl assessing the empirical support for this assumed connection between meditation and creativity, it is important to consider that creativity is not a unitary skill or process. Guilford (1950, 1967) suggested that creativity is composed of two main ingredients: divergent and convergent thinking - even though other processes are also likely to contribute (Wallas 1926).

Divergent thinking is taken to represent a style of thinking that allows many new ideas being generated, in a context where more than one solution is correct. An example of divergent thinking, and the associated creative flow (Csikszentmihalyi 1997), is improvisation (inventing, composing, or performing something with little or no preparation), an idea-generation technique used by actors and musician. In order to objectively measure the generation of new ideas, Guilford developed the so-called Alternate Uses Task (1967), in which participants are confronted with an everyday-use item, such as towel, and are supposed to list the different uses of that item, such as "waving it as a flag", "drying wet hair", and so on.

In contrast, convergent thinking is considered a process of identifying the "correct" and "unique" answer to a welldefined problem. Convergent thinking is heavily emphasized (often at the expense of divergent thinking) in standard educational systems, where students often face multiple-choice tests to find the right answer among a few alternatives. A wellestablished task to assess convergent thinking is Mednick's (1962) Remote Associates Task (RAT), in which participants are presented with three unrelated words, such as "cottage", "swiss", and "cake", and are to identify the common associate ("cheese"). Interestingly, Akbari Chermahini and Hommel (2010) found no correlation between performance on the Alternate Uses Task (AUT) and the RAT, supporting Guilford's (1967) speculation that convergent and divergent thinking represent separable components of human creativity.

The scientific evidence regarding the connection between meditation and creativity is very mixed (see Horan 2009, for a 
review). While some studies obtained evidence for a strong positive impact of meditation practice on creativity (Ball 1980; Orme-Johnson and Granieri 1977; Orme-Johnson et al. 1977), others found only a weak association or no effect at all (Cowger 1974; Domino 1977). Given the many differences between existing studies in terms of how creativity was assessed and how meditation was operationalized, it is difficult to say what the reasons for these discrepancies might be, but it seems clear that more theoretical guidance and conceptual clarity are needed.

In a recent study, Colzato et al. (2012) attempted to overcome the methodological and theoretical diversity across previous studies by distinguishing between convergent and divergent thinking (as assessed by the RAT and the AUT, respectively) and between focused-attention (FA) meditation and open-monitoring (OM) meditation, the two main techniques of Buddhist meditation (Lutz et al. 2008). In FA meditation, the individual focuses on a particular item, thought, or object. Everything else that might tend to attract attention, such as bodily sensations, environmental noise, or intrusive thoughts, is to be actively ignored by redirecting attention constantly back on the same focus point. In OM meditation, instead, the individual is free to attend any upcoming sensation or thought without any restriction, which requires attentional flexibility. Keeping in mind the distinction between FA and OM meditation, it is important to note that many exercises represent mixtures of these two types (Cahn and Polich 2006; Lippelt et al. 2014). It is also important to note that even if this article is based on the theoretical framework of distinguishing FA and OM, another one includes a new taxonomic system using an essentialist third-person approach within the domains of affect and cognition (Nash and Newberg 2013). These authors distinguish three Linnaeantype Domains: (a) affective-directed methods which induce an enhanced affective state during the meditation session (loving-kindness techniques), (b) null-directed methods which induce an empty non-cognitive/non-affective state (transcendental meditation techniques), and (c) cognitive-directed methods which engender an enhanced cognitive state (Samatha and Vipassana).

Colzato et al. (2012) suggested that, given the different characteristics of these two types of meditation, they are likely to induce different cognitive-control states, which again would be likely to affect convergent and divergent thinking in different ways. In particular, divergent thinking would likely require or benefit from a control state that provides a minimum of top-down control and local competition, so that the individual can easily and quickly "jump" from one thought to another in an only weakly guided fashion (Hommel 2012). If so, this kind of thinking should benefit from OM meditation and the weak, "distributed" control we assumed it to induce. In contrast, convergent thinking would be likely to benefit from a strong top-down bias (which constrains and directs the search process) and from strong local competition (as only one solution can be correct). If so, this kind of thinking should benefit from FA meditation and the more focused control style it was hypothesized to induce. As predicted, Colzato et al. (2012) found that, in a group of practitioners, OM meditation facilitated divergent thinking, as assessed by the AUT, but not convergent thinking. In contrast, FA meditation showed only a weak and statistically unreliable tendency to support convergent thinking, as assessed by the RAT. This failure to reach significance might have been due to a counteracting effect of positive mood, which increased significantly after both FA and OM meditation. Positive mood has been shown to correlate with both evidence of phasic increases in striatal dopaminergic supply and improvements in divergent think (Akbari Chermahini and Hommel 2012a). Conversely, engaging in divergent thinking was shown to induce more positive mood, while engaging in convergent thinking induces negative-going mood (Akbari Chermahini and Hommel 2012b). Moreover, cognitive top-down control (which is presumably more required for convergent than for divergent thinking: Fischer and Hommel 2012) has been demonstrated to suffer from positive mood and to become stronger with negative mood (van Steenbergen et al. 2010). Taken together, these findings suggest that positive mood better supports divergent thinking than convergent thinking and raise the possibility that positive mood might even impair convergent thinking.

The goal of the present study was to replicate and extend our previous findings (Colzato et al. 2012). In particular, we had three aims: first, by comparing a group of practitioners and a group of novices, we were interested to see whether the impact of FA meditation and OM meditation on creativity tasks depends on prior meditation experience. Second, we were interested to see whether, by using a more concentrative version of FA meditation technique, Colzato et al.'s (2012) failure to demonstrate a reliable effect of FA meditation on convergent thinking might have been due to using a too relaxing (and, thus, too much mood-enhancing) version of it. Third, we were interested to see whether and to what degree meditation would affect not only performance in convergentand divergent-thinking tasks but also the strategies used (Bowden et al. 2005): participants sometimes report to have deliberately and methodically tried out different possible answers until the correct solution came across (an analytical strategy) and sometimes that a solution suddenly popped into their awareness (an insight strategy leading to an "aha moment"). Note that insight and analytical strategies are unlikely to represent non-overlapping processes (Hommel 2012). While an analytical strategy clearly involves more top-down constraints on the cognitive search process than insight, it still has a search component that requires flexibly moving from one memory trace to the next, and while the insight strategy 
clearly involves more extensive and less constrained cognitive search than the analytical strategy, it still involves some constraints. It is these relative differences that we based our hypotheses on. Further studies have shown that these two strategies can change from trial to trial and are associated with different brain states (Jung-Beeman et al. 2004). Considering the characteristics of FA and OM meditation, it makes sense to assume that an analytical strategy in solving RAT items would be more consistent with a control state induced by FA meditation while the insight strategy would be more consistent with the control state induced by OM meditation. If so, all other things equal, the relative tendency to solve RAT items analytically should be more pronounced after FA meditation than after OM meditation.

\section{Method}

\section{Participants}

Forty healthy, native Dutch speakers (23 females and 17 males) constituted the two groups of 20 practitioners (average length of meditation practice in both $\mathrm{FA}$ and $\mathrm{OM}=3.3$ years; mean age $=43.7 ; \mathrm{IQ}=100$ ) and 20 novices (mean age $=42.5$; $\mathrm{IQ}=102)$. The group of practitioners were equally experienced in practicing FA (Samatha) and OM (Vipassana); none of them reported to practice FA or OM exclusively. Participants volunteered for the study without a financial reward, except partial compensation of travel expenses. Informed consent was obtained from all participants after the nature of the study was explained to them. The protocol was approved by the local ethical committee (Leiden University, Faculty of Social and Behavioral Sciences).

\section{Procedure}

Upon arrival to the lab, participants were asked to rate their mood. Next, the participant sat upright on a chair behind the computer where the testing would be done. Eyes were closed in OM condition. The same instructor, certified in Samatha, Mindfulness and Breathfulness training, provided the instruction for both sessions. Participants served in two 1-h sessions separated by 10 days. In one session, they performed under the supervision of a certified meditation instructor the FA meditation, rated their mood again, and completed our RAT version [compound Remote Associates Task (cRAT), see below] within $30 \mathrm{~min}$ and the AUT (Guilford 1967) within $2.5 \mathrm{~min}$. In the other session, the method was the same except that participants performed the OM meditation and completed new items of the cRAT and AUT. The order of session type and tasks was counterbalanced across participants: 10 participants performed first under OM and second under FA instruction, and another 10 performed first under FA and second under $\mathrm{OM}$ instruction. Five participants of each of these groups worked on the AUT followed by cRAT in their first session and on the cRAT followed by the AUT in their second session, while the other five participants of each group worked on the two tasks in reverse order. At the end of their second session, participants completed the Standard Progressive Matrices (SPM; Raven 1965). The cRAT and AUT were scored by two independent raters blinded to the experimental conditions.

\section{Measures}

\section{Focused-Attention Meditation}

The FA meditation took approximately 20 min, during which the participant was guided by a professional meditation instructor. Following Samatha tradition, the aim of which is to focus on a particular object, the participant uses a piece of wood that was placed on the table in front of him/her, to anchor and focus attention while resisting any distraction. The instructor verbally guided the participant by asking him/ her to direct attention to the object's features and to redirect attention to that same object when being distracted.

\section{Open-Monitoring Meditation}

Just like for the FA, the OM meditation session took approximately $20 \mathrm{~min}$, during which the participant was guided by a professional meditation instructor. The instructor used a method called Breathfulness ${ }^{\circledR}$ developed by Marco and Jacquelien de Jager (De Jager and De Jager 2013). This method incorporates the open-monitoring elements of the Buddhist Vipassana method of meditation into a harmonizing breath session, with a goal of reaching clarity and insight through seeing things "as they really are" without judgement or manipulation. Through cultivating a certain state of awareness of a connection between body and mind, via conscious breathing, one allows all objects of attention - endogenous or exogenous - to arise and be acknowledged as they are, without suppressing or resisting. The idea is that as one follows the rhythm of the breathing and accepts every rising mental experience, every breath brings a deeper awareness of initially hidden layers of one's being and eventually leads to transformation and clarity. The instructor verbally guided the participant by instructing him/her to breathe, to be open, and to observe instead of judge whatever thought or emotion might occur, facilitating an open-monitoring state.

Compound Remote Associates Task To obtain reliable estimates of strategy use in the RAT, Bowden and Jung-Beeman (2003) have recommended to use more items than the standard 20-30 items of the RAT (Akbari Chermahini and Hommel 2012a, b; Mednick 1962). Accordingly, we translated their extended item pool (called the compound Remote 
Associates Task; cRAT) into Dutch, as far as semantically possible, which left us with a pool of 220 items. Trials began with a central fixation cross $(1000 \mathrm{~ms})$ presented on a computer screen, followed by three problem words (such as French, car, shoe) presented simultaneously in horizontal orientation above, at, and below fixation. Participants are asked to find a compound word (horn) or two-word phrase with the solution word, which can come before or after any of the three words (within $10 \mathrm{~s}$ ). After giving the solution, participants were requested to identify which problem-solving strategies they used (analytical vs. insight). In each of the two sessions, participants completed 110 different items randomly selected for each participant after each meditation condition.

Alternate Uses Task In this task, participants were asked to list as many possible uses for common household items. In the two sessions, participants completed one different item (either brick or towel). The results can be scored in several ways with flexibility, the number of different categories used being the most consistent and reliable (Akbari Chermahini and Hommel 2010).

Flexibility - the number of different categories used.

Fluency - the total of all responses.

Originality - each response is compared to the total amount of responses from all of the subjects. Responses that were given by only $5 \%$ of the group count as unusual (1 point) and responses given by only $1 \%$ of them count as unique (2 points).

Elaboration - the amount of detail [e.g., "a doorstop" counts 0 , whereas "a door stop to prevent a door slamming shut in a strong wind" counts 2 (1 point for explanation of door slamming and another for further detail about the wind)].

Fluid Intelligence Individual fluid intelligence was estimated by a shortened, computerized version of the Raven's Standard Progressive Matrices (SPM; Raven 1965), as used in previous studies (e.g., Keizer et al. 2010). The SPM assesses the individual's ability to create perceptual relations and to reason by analogy independent of language and formal schooling; it is a standard, widely used test to measure Spearman's g factor as well as fluid intelligence (Raven 1965).

Affect Grid Mood and arousal were rated on a $9 \times 9$ pleasure $\times$ arousal grid (Russell et al. 1989), printed on paper, with values ranging from -4 to 4 .

\section{Statistical Analysis}

Independent $t$ tests were performed to test differences between the two meditation experience groups. From the two creativity tasks, six dependent variables were extracted for each participant: flexibility, fluency, originality, and elaboration scores from the Alternate Uses Task (AUT) [(all scored by two independent readers; Cronbach's alpha=0.99 (fluency); 0.94 (flexibility); 0.98 (originality); 0.93 (elaboration)] and the number of correct items from the cRAT, separated by strategy (analytical vs. insight). AUT measures were analyzed by means of repeated-measures ANOVAs with Session (OM vs. FA) as within-subjects factor and group (practitioners vs. novices) as between-group factor. cRAT scores were analyzed using the same ANOVA design above but with strategy (analytical vs. insight) as additional within-participants subject factor. Moreover, in order to rule out an order effect, AUT measures and cRAT scores were compared between OM-FA and FA-OM order conditions as a between-group factor. For the mood and arousal scores, time of measurement (pre- vs. post-meditation) was added as within-subjects factor.

A significance level of $p<0.05$ was adopted for all tests.

\section{Results}

No significant group differences were obtained for age $[t(38)=0.54, p=0.59)$ and IQ $(t(38)=0.99, p=0.33]$.

Mood significantly increased after the session, $F(1,38)=$ 26.19, $p<0.0001$, mean squared error $(\mathrm{MSE})=2.154, \eta^{2} p=$ $0.408(0.9$ vs. 2.1$)$. This effect was modulated by the type of meditation, $F(1,38)=7.67, p<0.01, \mathrm{MSE}=1.800, \eta^{2} p=0.168$ : the enhancement of mood was stronger for OM meditation $(0.8$ vs. 2.5$)$ than for FA mediation (0.9 vs. 1.5$)$. Arousal did not rise after the session, $F<1(0.4$ vs. 0.5$)$, but the level of arousal tended to be modulated by the type of meditation, $F(1,38)=4.04, p=0.052, \mathrm{MSE}=1.896, \eta^{2} p=0.096$ : arousal tended to decrease more after OM meditation (0.3 vs. 0.0$)$ compared to FA mediation (0.5 vs. 1.0).

Replicating earlier findings (Colzato et al. 2012), all four scores of the AUT showed an advantage for the OM session over the FA session. While this advantage was reliable for flexibility $\left[F(1,38)=5.56, p<0.05, \mathrm{MSE}=4.351, \eta^{2} p=0.128\right]$ and fluency $\left[F(1,38)=5.34, p<0.05, \mathrm{MSE}=12.824, \eta^{2}\right.$ $p=0.123]$, it did not reach significance for elaboration $\left[F(1,38)=1.49, p=0.23, \mathrm{MSE}=1.689, \eta^{2} p=0.039\right]$ and originality $(F<1)$, see Table 1 . No significant interaction of session on the four scores of the AUT and group was found, $F$ S $<1$, as indication that both practitioners and novices profit the same way from the OM meditation. Moreover, no significant interaction of order of conditions on the four scores of the AUT, session, and group was found, $F_{\mathrm{S}}<1$.

In the cRAT, participants used significantly more insight (33.8) than analytical (15.1) strategy to solve the problems, $F(1,38)=42.97, p<0.0001, \mathrm{MSE}=325.487, \eta^{2} p=0.531$. This main effect was modulated by a three-way interaction 
Table 1 Means and standard error (SE, in parentheses) for flexibility, fluency, originality, and elaboration scores from the Alternate Uses Task (AUT), the number of correct items from the compound Remote Associates Task (cRAT), separated for strategy (analytical vs. insight), as a function of group (practitioners vs. novices) and meditation [focused attention (FA) vs. open monitoring (OM)]

\begin{tabular}{lccccr}
\hline Variables & \multicolumn{3}{l}{ Practitioners } & & \multicolumn{2}{l}{ Novices } \\
\cline { 2 - 3 } \cline { 6 - 6 } & FA & OM & & FA & \multicolumn{1}{c}{ OM } \\
\hline AUT & & & & \\
Flexibility & $4.9(0.5)^{*}$ & $6.4(0.6)^{*}$ & & $5.6(0.5)$ & $6.3(0.6)$ \\
Fluency & $10.4(0.9)^{*}$ & $13.0(1.2)^{*}$ & & $12.2(0.9)$ & $13.3(1.2)$ \\
Originality & $6.2(4.6)$ & $6.5(4.1)$ & & $7.1(4.9)$ & $6.0(5.1)$ \\
Elaboration & $0.9(0.3)$ & $1.3(0.4)$ & & $0.7(0.3)$ & $1.1(0.4)$ \\
cRAT & & & & $16.8(2.3)$ \\
Analytical & $15.2(2.6)$ & $11.6(2.3)$ & & $16.8(2.6)$ & 16.8 \\
Insight & $33.5(2.4)^{*}$ & $40.0(2.7)^{*}$ & & $31.9(2.4)$ & $29.8(2.7)$ \\
\hline
\end{tabular}

${ }^{*} p<0.05$

involving group and meditation, $F(1,38)=4.38, p<0.05$, MSE $=87.805, \eta^{2} p=0.103$. Separate ANOVAs per group revealed that the meditation and strategy interaction was reliable for practitioners, $F(1,19)=6.63, p<0.05, \mathrm{MSE}=78.489$, $\eta^{2} p=0.259$, but not for novices, $F<1$. Post-hoc multiple comparisons tests (Newman-Keuls) revealed that practitioners did not solve more cRAT items analytically after FA meditation than after OM meditation $(p=0.21)$, but solved more cRAT items with insight after OM meditation than after FA meditation $(p=0.03)$; see Table 1 . Moreover, no significant interaction of order of conditions on the cRAT scores, strategy used, session, and group was found, $F \mathrm{~S}<1$.

\section{Discussion}

The goal of the present study was to replicate and extend the previous findings of Colzato et al. (2012) showing that, in a group of practitioners, OM meditation facilitated divergent thinking but not convergent thinking, while FA meditation practice only showed an unreliable tendency going into the opposite direction. We modified the previous design in three important ways: we added a group of novices to see whether practice plays a role, tried to avoid possible mood artifacts by making the FA meditation more neutral (by attracting attention to a wooden block instead of the participant's own body), and assessed the strategy use in the convergent-thinking task to see whether meditation would affect the probability of solving items analytically or through insight.

As suggested by Capurso et al. (2014), our first question was whether the impact of meditation on creativity would depend on prior meditation experience, which raises the question whether and how much practice is necessary to obtain meditation effects on creativity and whether this effect is due to a trait (of being an individual interested in meditation), a state (ad hoc induced by meditation), or both. With the exception of a strategy effect to be more elaborated below, it seems that long-term meditation training (as opposed to one exposure) promotes more insight problem solving when involved in convergent thinking. In contrast, meditation training does not provide long-term benefits for divergent thinking. Not only could we replicate Colzato et al.'s (2012) observation that divergent thinking benefits more from OM meditation than from FA meditation, but we also found the same effect in novices. This suggests that, at least with the OM meditation technique we considered, prior practice is not a necessary requirement for meditation to impact creativity. Possibly, engaging in the meditation creates a particular mind state that supports or engages some cognitive processes and interferes with others.

Our second question was whether Colzato et al.'s (2012) failure to demonstrate reliable facilitation of convergent thinking by FA meditation might have been due to a too moodenhancing FA meditation technique. We tested that by using a more concentrative version of the FA meditation technique. This modification was partially successful: mood was still enhanced after the FA meditation, but the enhancement was smaller than after the OM meditation. Even though this does not rule out counteracting mood effects entirely, the overall pattern does not support the idea that FA meditation improves analytical thinking in general. This is very clear in novices, who show almost identical cRAT performance after the two meditations. And even in the practitioners, who do show meditation effects, the outcome suggests that the main action is related to OM meditation but not FA meditation-where practitioners show performance comparable to the novices. Hence, taken altogether, the present study supports the observation of Colzato et al. (2012) that divergent thinking is much better supported by OM meditation than convergent thinking is supported by FA meditation.

Our third question was whether meditation would affect the strategies used to find correct solutions in the convergentthinking task. To answer that question, we not only looked into general performance in our version of the RAT but also distinguished between items that were solved by means of an analytical strategy and items that were solved by insight (Bowden et al. 2005). We considered that FA meditation might favor an analytical strategy while OM meditation might favor insight solutions. The outcome is partially consistent with this expectation, but the effect was restricted to OM meditation and only found in practitioners. As Table 1 shows, performance is comparable for novices after both meditation conditions and practitioners after FA meditation, while practitioners after OM meditation show a strong shift from analytical to insight solutions, which under this combination account for about $75 \%$ of all correct solution. 
We can only speculate what the reasons for this outcome pattern are. It could be that those that have chosen to engage in OM meditation (practitioners, that is) have a particular preference for mental states that are less top-down controlled, or better abilities to reach them. If so, simply giving novices more training may not necessarily produce the same outcome. For instance, individuals with a genetic predisposition that favors divergent thinking might be drawn to OM meditation, so that what looks like an effect of practice might actually represent a kind of self-selection.

Another possibility is to interpret our results in terms of what Lutz et al. (2008) described as "effortful" or "grasping" states versus "effortless" or "non-grasping" states. Effortful states might not induce positive mood to the same degree as effortless states do because increased effort, or cognitive demand, is likely to impair mood (Akbari Chermahini and Hommel 2012b). The idea that OM might induce effortless or non-grasping states fits well with observation that $\mathrm{OM}$ promotes divergent thinking, whereas FA, which might promote effortful states, does not promote convergent thinking.

To summarize, we found that meditation techniques have specific effects on the cognitive processes involved in creativity tasks. In our study, OM meditation seemed to be more effective than FA meditation. Engaging in OM meditation (as compared to FA meditation) yielded better performance in divergent thinking, irrespective of previous experience or training. This suggests that OM meditation induces a temporary mind state that reduces top-down control and allows for a more associative spreading of activation in memory. This also fits with the observation that OM meditation impaired truly analytical thinking in the convergent-thinking task while supporting alternative, less analytical search strategies. FA meditation was clearly less effective and did not produce a systematic positive impact on either convergent or divergent thinking.

Future studies need to address how short-lived the effect of meditation on creativity might be, that is, whether the positive effect is restricted only to performance directly after meditating. Moreover, individual differences would also need to be taken into account in explaining the relationship between the two different types of meditation and creativity performance. If we consider meditation a form of cognitive training, such as videogame practice (Colzato et al. 2013), individual differences may affect the degree to which individuals can benefit from meditating: individuals with certain genetic predispositions may take advantage from OM meditation, whereas individuals with another predisposition may benefit from FA meditation or other sorts of meditative techniques.

Acknowledgments The research of L.S. Colzato is supported by a Vidi grant (\#452-12-001) of the Netherlands Organization for Scientific Research (NWO).

\section{References}

Akbari Chermahini, S., \& Hommel, B. (2010). The (b)link between creativity and dopamine: spontaneous eye blink rates predict and dissociate divergent and convergent thinking. Cognition, 115, 458465.

Akbari Chermahini, S., \& Hommel, B. (2012a). More creative through positive mood? Not everyone! Frontiers in Human Neuroscience, 6 , 319.

Akbari Chermahini, S., \& Hommel, B. (2012b). Creative mood swings: divergent and convergent thinking affect mood in opposite ways. Psychological Research, 76, 634-640.

Ball, O. E. (1980). The effect of TM and the TM-Sidhi program on verbal and figural creativity (TTCT), auditory creativity (SandI), and hemispheric dominance (SOLAT). Unpublished doctoral dissertation, University of Georgia.

Bowden, E. M., \& Jung-Beeman, M. (2003). One hundred forty-four compound remote associate problems: short insight-like problems with one-word solutions. Behavioral Research, Methods, Instruments, and Computers, 35, 634-639.

Bowden, E. M., Jung-Beeman, M., Fleck, J., \& Kounios, J. (2005). New approaches to demystifying insight. Trends in Cognitive Sciences, 9, 322-328.

Cahn, B. R., \& Polich, J. (2006). Meditation states and traits: EEG, ERP, and neuroimaging studies. Psychological Bulletin, 132(2), 180-211.

Capurso, V., Fabbro, F., \& Crescentini, C. (2014). Mindful creativity: the influence of mindfulness meditation on creative thinking. Frontiers in Psychology. doi:10.3389/fpsyg.2013.01020.

Colzato, L. S., Ozturk, A., \& Hommel, B. (2012). Meditate to create: the impact of focused-attention and open-monitoring training on convergent and divergent thinking. Frontiers in Psychology, 3, 116.

Colzato, L. S., van den Wildenberg, W. P., \& Hommel, B. (2013). Cognitive control and the COMT Val158Met polymorphism: genetic modulation of videogame training and transfer to task-switching efficiency. Psychological Research, 1-9.

Cowger, E. L. (1974). The effects of meditation (zazen) upon selected dimensions of personality development. Dissertation Abstracts International, 34, 8-A, Part 1, 4734.

Csikszentmihalyi, M. (1997). Flow and the psychology of discovery and invention. New York: HarperPerennial.

De Jager, M., \& De Jager, J. (2013). Breathfulness: Als weg naar een verruimd leven. Steenbergen: InsideOut. (ISBN 978.908.1194.303).

Domino, G. (1977). Transcendental meditation and creativity: an empirical investigation. Journal of Applied Psychology, 62, 358-362.

Fischer, R., \& Hommel, B. (2012). Deep thinking increases task-set shielding and reduces shifting flexibility in dual-task performance. Cognition, 123, 303-307.

Guilford, J. P. (1950). Creativity. American Psychologist, 5, 444- 454.

Guilford, J. P. (1967). The nature of human intelligence. New York: McGraw-Hill.

Hommel, B. (2012). Convergent and divergent operations in cognitive search. In M. Todd, T. T. Hills, \& T. W. Robbins (Eds.), Cognitive search: evolution, algorithms, and the brain. Strüngmann Forum reports (Vol. 9, pp. 215-230). Cambridge: MIT Press.

Horan, R. (2009). The neuropsychological connection between creativity and meditation. Creativity Research Journal, 21(2-3), 199-222.

Isaacson, W. (2011). iSteve: The book of Jobs. Simon \& Schuster (U.S.). (ISBN 1.4516.4853.7).

Jung-Beeman, M., Bowden, E. M., Haberman, J., Frymiare, J. L., Arambel-Liu, S., Greenblatt, R., Reber, P. J., \& Kounios, J. (2004). Neural activity observed in people solving verbal problems with insight. Public Library of Science - Biology, 2, 500-510.

Keizer, A. W., Verschoor, M., Verment, R., \& Hommel, B. (2010). The effect of gamma enhancing neurofeedback on measures of feature- 
binding flexibility and intelligence. International Journal of Psychophysiology, 75, 25-32.

Lippelt, D. P., Hommel, B., \& Colzato, L. S. (2014). Focused attention, open monitoring and loving kindness meditation: effects on attention, conflict monitoring and creativity. Frontiers in Psychology, 5, 1083. doi:10.3389/fpsyg.2014.01083.

Lutz, A., Slagter, H. A., Dunne, J. D., \& Davidson, R. J. (2008). Attention regulation and monitoring in meditation. Trends in Cognitive Sciences, 12(4), 163-169.

Mednick, S. (1962). The associative basis of creative problem solving process. Psychological Review, 69, 200-232.

Nash, J. D., \& Newberg, A. (2013). Toward a unifying taxonomy and definition for meditation. Frontiers in Psychology, 4, 806.

Orme-Johnson, D. W., \& Granieri, B. (1977). The effects of the age of enlightenment governor training courses on field independence, creativity, intelligence, and behavioral flexibility. In D. W. OrmeJohnson \& J. T. Farrow (Eds.), Scientific research on Maharishi's
Transcendental Meditation and TM-Sidhi Program, collected papers (Vol. 1, pp. 713-718). New York: MERU Press.

Orme-Johnson, D. W., Clements, G., Haynes, C. T., \& Badaoui, K. (1977). Higher states of consciousness: EEG coherence, creativity, and experiences of the siddhis. In D. W. Orme-Johnson \& J. T. Farrow (Eds.), Scientific research on Maharishi's Transcendental Meditation and TM-Sidhi Program, collected papers (Vol. 1, pp. 705-712). New York: MERU Press.

Raven, J. C. (1965). Advanced progressive matrices set I and II. London: H.K. Lewis.

Russell, J. A., Weis, A., \& Mendelsohn, G. A. (1989). Affect grid: a single-item scale of pleasure and arousal. Journal of Personality and Social Psychology, 57, 493-502.

van Steenbergen, H., Band, G. P. H., \& Hommel, B. (2010). In the mood for adaptation: how affect regulates conflict-driven control. Psychological Science, 21, 1629-1634.

Wallas, G. (1926). The art of thought. New York: Harcourt Brace. 